\title{
The peculiar B-type supergiant HD 327083*
}

\author{
M. A. D. Machado ${ }^{1}$, F. X. de Araújo ${ }^{2}$, and S. Lorenz-Martins ${ }^{1}$ \\ 1 Observatorio do Valongo/UFRJ, Ladeira Pedro Antonio 43, 20080-090, Rio de Janeiro, Brazil \\ 2 Observatorio Nacional/MCT, Rua Gal. Jose Cristino 77, 20921-400, Rio de Janeiro, Brazil
}

Received 31 October 2000 / Accepted 4 February 2001

\begin{abstract}
Coudé spectroscopic data of a poorly-studied peculiar supergiant, HD 327083, are presented. H $\alpha$ and $\mathrm{H} \beta$ line profiles have been fitted employing a non-LTE code adequate for spherically expanding atmospheres. Line fits lead to estimates of physical parameters. These parameters suggest that HD 327083 may be close to the Luminous Blue Variable phase but it is also possible that it could be a B[e] Supergiant.
\end{abstract}

Key words. stars: individual - stars: LBVs - stars: B $[\mathrm{e}]$ - evolutionary phases

\section{Introduction}

Luminous emission-line B-type stars often present massive winds. Many of them share some common properties: i) optical spectra dominated by $\mathrm{HI}$ and FeII emission lines, usually displaying P-Cygni profiles; ii) presence of forbidden emission lines, specially of [FeII]; iii) strong red/infrared excess. Such properties are typical of the B[e] Supergiants (Lamers et al. 1998) but are also often seen in the Luminous Blue Variables (LBVs). Their localization in the $\mathrm{H}-\mathrm{R}$ diagram is a real problem. The distances are very uncertain (particularly for galactic objects) and the absence of photospheric lines renders the assignment of a spectral type quite difficult.

The present paper concerns one of such interesting stars, namely HD 327083. Almost no study exists in the literature devoted to it, but some works on emissionline objects have included it. $\mathrm{H} \alpha$ emission was discovered by Merrill \& Burwell (1949). Carlson \& Henize (1979) included HD 327083 in their list of peculiar southern emission-line objects. They have proposed a B8 spectral class. Some years ago Lopes et al. (1992) showed observations of some spectral regions. They revealed HI lines with P-Cygni profiles as well as FeII $\lambda \lambda$ 4924, 5018, $5169 \AA$ (m42). On the other hand the FeII $\lambda$ transition at $9997 \AA$ seemed to be in pure emission. The authors have suggested a B6 type. More recently, Thé et al. (1994) presented a new catalogue of (confirmed and candidates) Herbig AeBe stars. In this catalogue HD 327083 is cited among the ex-

Send offprint requests to: F. X. Araujo, e-mail: araujo@on.br

* Based on observations made at Observatorio do Pico dos Dias, operated by MCT/Laboratorio Nacional de Astrofisica, Brazil. treme emission-line objects, which are not identified to belong to a certain group. However, they proposed the spectral type B1.5. The same assignment has been used by Valenti et al. (2000) while Sheikina et al. (1999) have obtained $\log T_{\text {eff }} \geq 4.2$ and $\log \frac{L}{L_{\odot}} \sim 6.0$.

Here we show our recent spectroscopic data and estimates of some physical parameters (as $\dot{M}, L_{\star}, T_{\star}$ and $A_{\mathrm{He}}$, the numerical abundance ratio $\left.n(\mathrm{He}) / n(\mathrm{H})\right)$. $\mathrm{H} \alpha$ and $\mathrm{H} \beta$ line profiles have been fitted with this aim. These estimates allow us to locate (within certain uncertainties) HD 327083 in the H-R diagram. A discussion of its evolutionary status, based on the derived parameters, is also presented.

\section{The data}

The spectra were obtained in August, 1999 using a Coudé spectrograph at the LNA $1.6 \mathrm{~m}$ telescope in Pico dos Dias (Brazil). A grating of $600 \mathrm{l} / \mathrm{mm}$ combined with a $1024 \times 1024$ CCD have been used; the reciprocal dispersion is about $0.4 \AA$ pixel $^{-1}$ yielding a wavelength range of $\sim 400 \AA$. The central wavelengths are: $\lambda_{\mathrm{c}}=4750$, 5000,5200 and $6400 \AA$. The data have been reduced using IRAF routines. We followed standard procedures: bias subtraction, flat-fielding, linearization, wavelength calibration and correction for atmospheric extinction.

In Figs. 1, 2 we can see our normalized spectra. In fact the spectral regions covered by our observations are dominated - besides $\mathrm{H} \alpha$ and $\mathrm{H} \beta$-, by FeII emission lines. Some of these lines show a P-Cygni profile and others are in pure emission. Table 1 lists the FeII transitions identified, which are marked in Figs. 1, 2. Column 1 gives the measured wavelength $(\AA)$ of emission peaks, followed by 

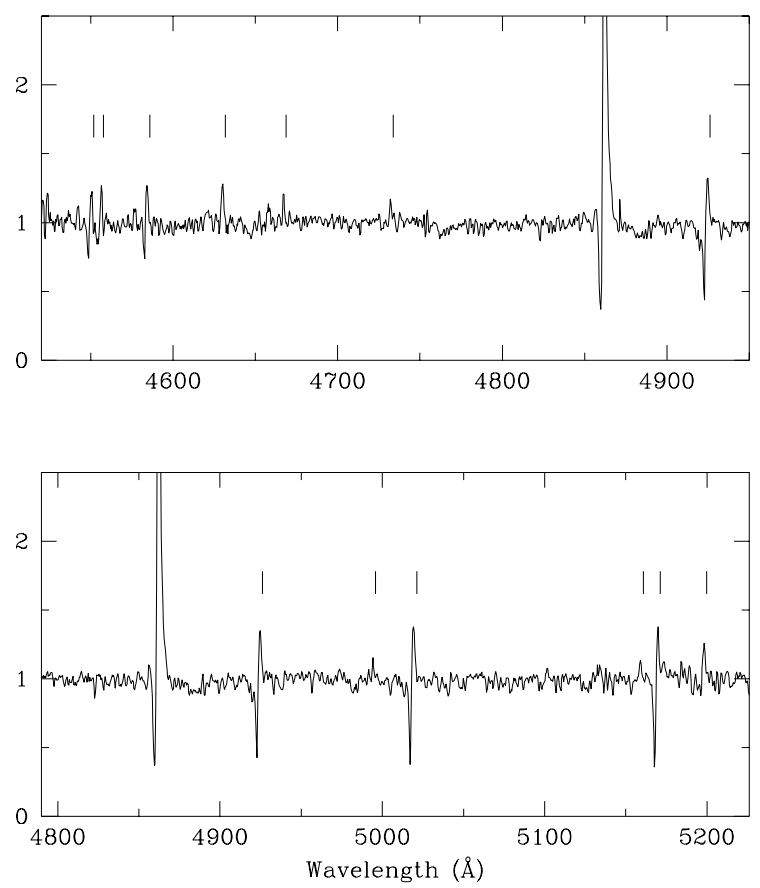

Fig. 1. Normalized spectra: $\lambda_{\mathrm{c}}=4750,5000 \AA$ (see text)
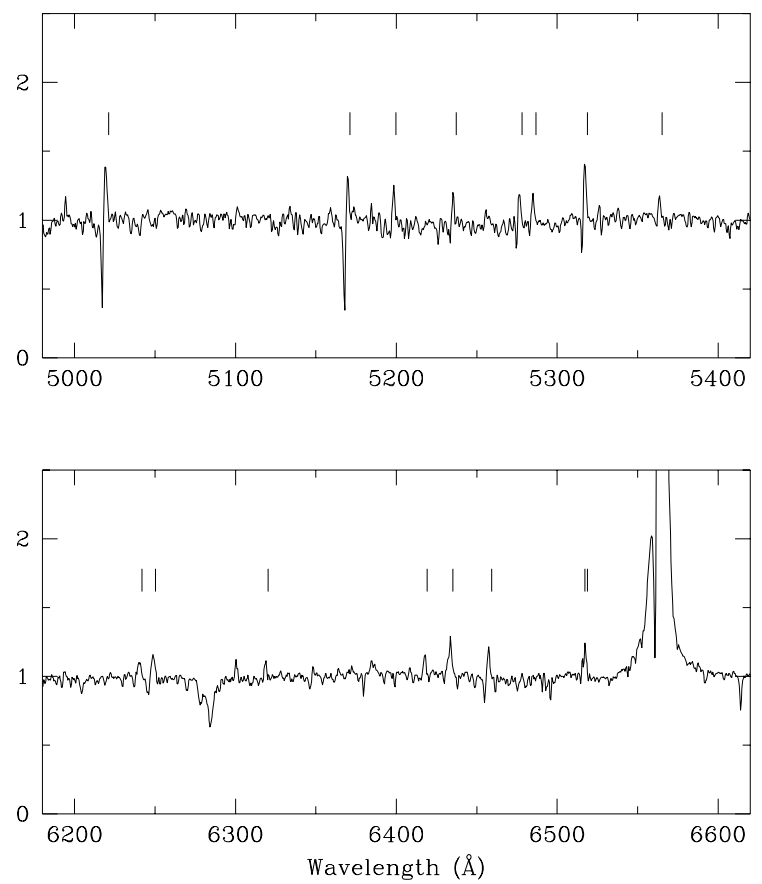

Fig. 2. Normalized spectra: $\lambda_{\mathrm{c}}=5200,6400 \AA$ (see text).

our identification (Col. 2). We note that only one feature, at $\lambda 5159.0 \AA$, has been recognized as a forbidden transition (see Fig. 1b). On the other hand [OI] $\lambda 6300.2 \AA$ is present (Fig. 2b).
Table 1. FeII lines

\begin{tabular}{|l|c|}
\hline Wavelength & Identification \\
\hline 4550.0 & $4549.5(\mathrm{~m} 38)$ \\
4556.0 & $4555.8(\mathrm{~m} 37)$ \\
4584.0 & $4582.8(\mathrm{~m} 37)$ \\
& $4583.8(\mathrm{~m} 38)$ \\
& $4584.0(\mathrm{~m} 26)$ \\
4630.0 & $4629.4(\mathrm{~m} 37)$ \\
4667.0 & $4666.8(\mathrm{~m} 37)$ \\
4732.0 & $4731.4(\mathrm{~m} 43)$ \\
4924.5 & $4923.9(\mathrm{~m} 42)$ \\
4994.0 & $4993.4(\mathrm{~m} 36)$ \\
5019.5 & $5018.4(\mathrm{~m} 42)$ \\
5159.0 & $5158.0(18 \mathrm{~F}]$ \\
& $5158.8(19 \mathrm{~F}]$ \\
5169.5 & $5169.0(\mathrm{~m} 42)$ \\
5198.0 & $5197.6(\mathrm{~m} 49)$ \\
5235.5 & $5234.6(\mathrm{~m} 49)$ \\
5276.5 & $5276.0(\mathrm{~m} 49)$ \\
5285.0 & $5284.1(\mathrm{~m} 41)$ \\
5317.0 & $5316.6(\mathrm{~m} 49)$ \\
& $5318.8(\mathrm{~m} 48)$ \\
5363.5 & $5362.9(\mathrm{~m} 48)$ \\
6240.0 & $6238.2(\mathrm{~m} 74)$ \\
& $6239.4(\mathrm{~m} 34)$ \\
6248.5 & $6247.6(\mathrm{~m} 74)$ \\
6318.5 & $6318.0\left(\mathrm{z}^{4} \mathrm{D}{ }^{\circ}-\mathrm{c}^{4} \mathrm{D}\right)$ \\
6417.5 & $6417.9(\mathrm{~m} 74)$ \\
6433.5 & $6432.7(\mathrm{~m} 40)$ \\
6457.5 & $6456.4(\mathrm{~m} 74)$ \\
6517.0 & $6518.2(\mathrm{~m} 72)$ \\
\hline
\end{tabular}

\section{Summary of the model}

Let us briefly describe now the model employed. Recently Machado (1998, 1999) developed a non-LTE numerical code for radiation transfer in expanding stellar atmospheres of massive stars. This code is based on basic assumptions: spherical symmetry, stationarity and homogeneity. The density structure is related to the mass loss rate and the velocity field $V(r)$ via the equation of continuity

$\rho(r)=\frac{\dot{M}}{4 \pi r^{2} V(r)}$

with $\dot{M}$ as an input parameter and the velocity field is pre-specified in an ad-hoc way as a $\beta$-type law

$V(r)=V_{\infty}\left(1-R_{\star} / r\right)^{\beta}$

where $V_{\infty}$ is the terminal velocity of the wind and $\beta$ is a measure of the rate of acceleration (de Koter et al. 1993).

The code is based on the following scheme. The statistical equilibrium equations are solved using the escape probability method for calculating the source function while the transfer equation is solved using the "SEI" Sobolev Exact Integration - method (Lamers et al. 1987). This is justified by the flow velocities observed in the wind of HD 327083. 
In the present version it is considered that the wind begins in the sonic point localized at radius $R_{\star}$. The effective temperature $T_{\star}$ is defined by the luminosity $L_{\star}$ and the radius $R_{\star}$ via the Stefan-Boltzmann law.

Regarding the temperature structure we have used the law suggested by Drew (1985):

$T(r)=T_{\star}\left[0.78-\left(0.51 \frac{V(r)}{V_{\infty}}\right)\right]$.

We are aware that this temperature profile is more appropriate to main sequence $\mathrm{O}$ stars. Thus we have checked the influence of the temperature law. Some simple tests with isothermal and radially decreasing ad-hoc profiles have been performed. It was found that the final result is not sensitive to similar temperature laws.

The model atmosphere in the present work consists of hydrogen and helium. For $\mathrm{H}$ and HeII we consider the lowest ten bound levels. For HeI we adopt the atomic model suggested by Almog \& Netzer (1989) that includes the individual levels until $n \leq 4$ and combined levels with $5 \leq n \leq 10$.

The determination of the population numbers of the energy levels is made in a non-LTE way solving the equations of statistical equilibrium for each level considered in the atomic model. The radiative transition probabilities and the photoionization cross section for all levels considered are quoted from the Opacity Project.

The ionization collision cross sections have been obtained from the approximate formulae given by Jefferies (1968). Excitation collisional rates have been calculated from the collisional strength values. For $\mathrm{H}$ and $\mathrm{HeII}$ these values are computed using polynomials given by Giovanardi et al. (1987). For HeI the collision strengths are taken from Berrington \& Kingston (1987) for levels with $n \leq 4$ and from Auer \& Mihalas (1968) and Mihalas $\&$ Stone (1968) to the other ones.

The radiation and the statistical equation systems are solved simultaneously through the wind that is divided in shells. A convergence criterium is applied in order to move from one shell to the next.

\section{4. $\mathrm{H} \alpha$ and $\mathrm{H} \beta$ line fits}

\subsection{Methodology}

The input parameters are the temperature $T_{\star}$, the stellar radius $R_{\star}$ (and luminosity), the mass loss rate $\dot{M}$, the terminal velocity $V_{\infty}$, the helium abundance $A_{\mathrm{He}}$ and the free parameter of the velocity profile $\beta$. In the present work we have fixed the value of the terminal velocity, $V_{\infty}=400 \mathrm{kms}^{-1}$. This value is an average of the $V_{\text {edges }}$ of $\mathrm{H} \alpha$ and $\mathrm{H} \beta$ profiles.

Due to the great number of "free" parameters we have adopted the following strategy in order to obtain the line fits. First we defined temperature limits. Previous determinations of temperature for HD 327083 are very uncertain. As discussed in the introduction first estimates have pointed to values around $T_{\text {eff }} \sim 11000 \mathrm{~K}-13000 \mathrm{~K}$.
More recent ones have lead to larger values: $T_{\text {eff }} \sim$ $16000 \mathrm{~K}-19000 \mathrm{~K}$. So we have decided to explore a broad range, that roughly corresponds to the LBVs and Yellow Supergiants domain: $8000 \mathrm{~K}-31000 \mathrm{~K}$. This $T_{\text {eff }}$ range was fixed based on the works by Gummersbach et al. (1995); Szeifert et al. (1996); de Jager \& Nieuwenhuijzen (1997).

Secondly we have started computing models according to the points of the evolutionary tracks by Schaller et al. (1992) from $M_{\mathrm{ZAMS}}=20 M_{\odot}$ to $120 M_{\odot}$. Each track consists of a number of points which provide stellar temperature, luminosity, mass loss rate and surface abundance ratio $\left(A_{\mathrm{He}}\right)$. These are the entry parameters for the code. The evolutionary points which give reasonably good fits (according to a $\chi^{2}$ criterium) are picked as starting points. From them we subsequently varied the input parameters, specially mass loss, in order to obtain better adjustments.

\subsection{Results}

The mass loss rate is indeed small for the $M_{\text {ZAMS }}=20 M_{\odot}$ and $M_{\mathrm{ZAMS}}=25 M_{\odot}$ paths. It is not enough to produce $\mathrm{H} \alpha$ and $\mathrm{H} \beta$ profiles with the intensities observed in HD 327083. Concerning the $M_{\mathrm{ZAMS}}=85 M_{\odot}$ and the $M_{\text {ZAMS }}=120 M_{\odot}$ tracks, in such cases the He abundance is already high for stars just evolved off the Main Sequence. In these regions the temperatures are high $\left(T_{\star} \geq 40000 \mathrm{~K}\right)$ and appropriate fits of $\mathrm{H} \alpha$ and $\mathrm{H} \beta$ cannot be achieved with reasonable values for the others parameters.

Our best fits have been obtained with the following set of parameters. Model 1: $\dot{M}=4.910^{-5} M_{\odot} \mathrm{yr}^{-1}$, $T_{\star}=19000 \mathrm{~K}, L_{\star}=510^{5} L_{\odot}, A_{\mathrm{He}}=0.40$. Model 2 : $\dot{M}=1.010^{-4} M_{\odot} \mathrm{yr}^{-1}, T_{\star}=9000 \mathrm{~K}, L_{\star}=9.510^{5} L_{\odot}$, $A_{\mathrm{He}}=0.11$. These fits are shown in Figs. $3 \mathrm{a}$ and $3 \mathrm{~b}$ respectively and were both obtained with $\beta=3$, which indicates a slow expansion. Model 1 is based on the series of $\mathrm{H} \alpha$ and $\mathrm{H} \beta$ profiles along the $M_{\mathrm{ZAMS}}=40 M_{\odot}$ path while model 2 is based on the $M_{\mathrm{ZAMS}}=60 M_{\odot}$ path. They have been selected following the methodology described earlier. We note that the "best model" values of $\dot{M}$ are slightly higher (about $50 \%$ ) than those given directly by the evolutionary paths.

It can be seen that the agreement between observational and theoretical profiles is rather poor. The models reproduce well the emission strengths but the absorption features are quite flat and narrow. This could suggest that the emission originates in a high density disk. Unfortunately we cannot check this alternative within the scope of our spherical model.

\section{Discussion}

Each set of parameters leads to a location in the H-R diagram. Figure 4 shows these positions and some evolutionary paths. Two alternatives - redward or blueward - are possible if only $T_{\star}$ and $L_{\star}$ are considered. He abundance may be invoked to choose one possibility or another. 

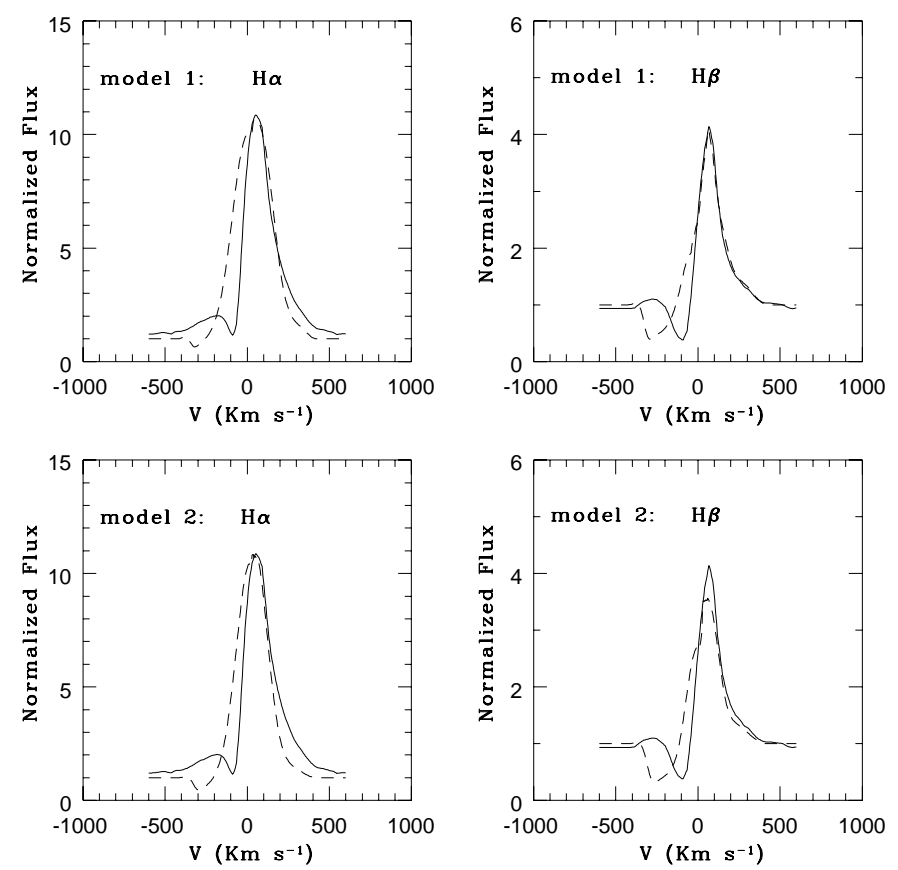

Fig. 3. Observational data (dashed lines) and best theoretical fits (full lines) for $\mathrm{H} \alpha$ and $\mathrm{H} \beta$ profiles

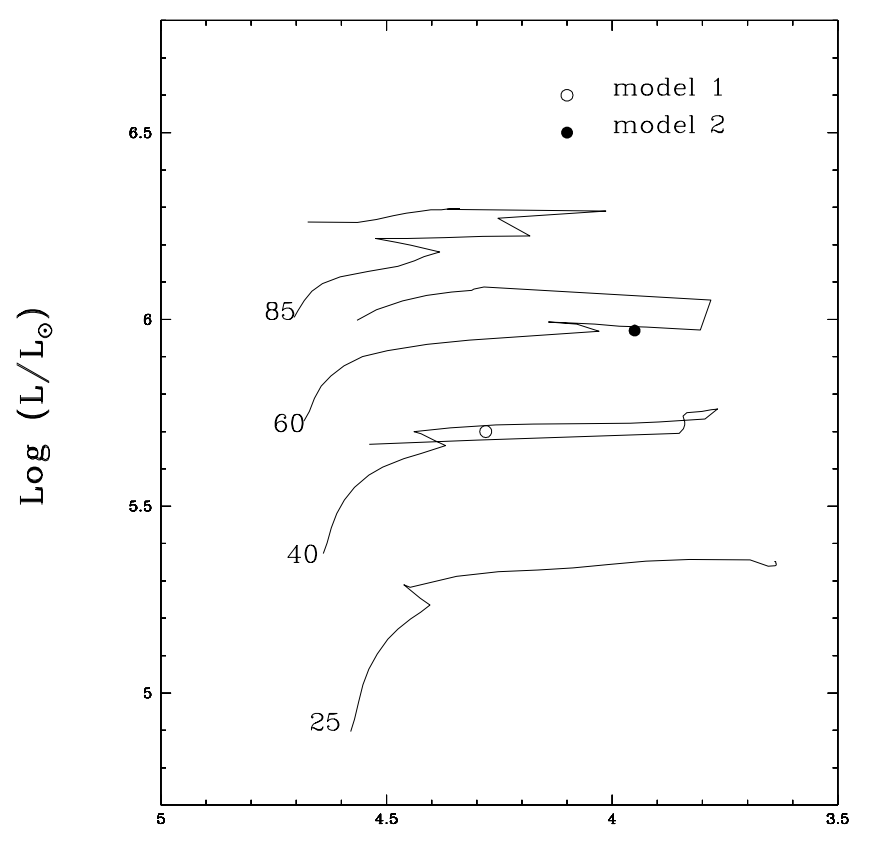

$\log \mathrm{T}_{\text {eff }}$

Fig. 4. Theoretical evolutionary tracks of $25 M_{\odot} ; 40 M_{\odot}$; $60 M_{\odot}$ and $85 M_{\odot}$ initial main-sequence mass. The circles locate the best model parameters

The evolutionary model 1 has $A_{\mathrm{He}}=0.40$. This value is in the blueward track: the star would be coming back to the hotter region of the diagram. The parameters in this scenario point to a LBV quiescent (or post-LBV) phase. On the other hand the evolutionary model 2 has $A_{\mathrm{He}}=0.11$. This low value indicates a point in the red- ward track. Here the star is in a less evolved stage, coming to the LBV eruptive phase. The intensity of the He lines should, in principle, reflect the He abundance. He lines are not seen in our data. We note however that our spectra do not cover the wavelength regions with strong He lines, such as $\lambda=5876,6678 \AA$. So, we prefer not to discard model 1 at present time.

Both alternatives allow us to propose that HD 327083 is near the LBV phase. However, this suggestion must be taken with caution. First because we have fitted only $\mathrm{H} \alpha$ and $\mathrm{H} \beta$ line profiles. So, the uncertainty in the derived parameters is large. Second because the high value of $\beta$ needed to obtain "good" fits reinforces the scenario of emission coming mostly from a flattened region, as the equatorial disk believed to be present in B[e] Supergiants (Zickgraf et al. 1986).

In general the $\mathrm{B}[\mathrm{e}]$ stars have stronger $[\mathrm{FeII}]$ lines than the LBVs. In our spectra only the feature at $\lambda 5159.0 \AA$ (which we attribute to transitions in multiplets $[18 \mathrm{~F}]$ and $[19 \mathrm{~F}]$ ) seems to be present in Fig. 1b, although it is uncertain in Fig. 2a. It is true that our data only covers the regions $\lambda \sim 4550-5400 \AA$ and $\lambda \sim 6200-6000 \AA$. However, several forbidden transitions (from multiplets $[3 \mathrm{~F}],[4 \mathrm{~F}]$, $[18 \mathrm{~F}],[19 \mathrm{~F}],[35 \mathrm{~F}]$ etc.) are in these regions. On the other hand $[\mathrm{OI}] \lambda 6300.2 \AA$ is clearly seen (Fig. 2b). This feature is intense in the $\mathrm{B}[\mathrm{e}]$ Supergiants but absent in the LBVs.

It is likely that HD 327083 is in a short-lived evolved stage of massive stars. However it is still unclear if it is a $\mathrm{LBV}$ candidate or if it is a $\mathrm{B}[\mathrm{e}]$ Supergiant. It is sure that it deserves to be further investigated. Such analysis should be based on higher-quality spectroscopic data, covering a broad spectral domain. Moreover it should include other information about the star such as the energy distribution, Balmer jump, and colour. From the theoretical point of view it is needed to employ a non-spherical code (e.g. Stee et al. 1995) to obtain adequate fittings not only for HI lines but also for HeI and $\mathrm{N}$ lines. This detailed investigation will clarify the nature of HD 327083 and it may contribute to a better understanding of evolved massive stars.

Acknowledgements. The authors thank the questions and comments of the referee, $\mathrm{H}$. Lamers, which greatly improved this paper. M. Machado and S. Lorenz Martins acknowledge CNPq (300320/99-0) and FUJB (FUJB 8635-5), respectively, for financial support.

\section{References}

Almog, Y., \& Netzer, H. 1989, MNRAS, 238, 51

Auer, L. H., \& Mihalas, D. 1968, ApJ, 151, 311

Berrington, K. A., \& Kingston, A. E. 1987, J. Phys. B, 20, 6631

Carlson, E. D., \& Henize, K. G. 1979, Vistas Astron., 23, 213 de Jager, C., \& Nieuwenhuijzen, H. 1997, MNRAS, 290, 250

de Koter, A., Schmutz, W., \& Lamers, H. J. G. L. M. 1993, A\&A, 277, 561

Drew, J. E. 1985, MNRAS, 217, 867

Giovanardi, C., Natta, A., \& Palla, F. 1987, A\&AS, 70, 269 
Gummersbach, C. A., Zickgraf, F.-J., \& Wolf, B. 1995, A\&A, 302, 409

Jefferies, J. T. 1968, in Spectral Line Formation (Blaisdell Publish. Co., Waltham-Massachussets)

Lamers, H. J. G. L. M., Cerruti-Sola, M., \& Perinoto, M. 1987, ApJ, 314, 762

Lamers, H. J. G. L. M., Zickgraf, F. J., de Winter, D., Houziaux, L., \& Zorec, J. 1998, A\&A, 340, 117

Lopes, D. F., Daminelli Neto, A., \& de Freitas Pacheco, J. A. 1992, A\&A, 261, 482

Machado, M. 1998, in Determinação de parâmetros estelares de objetos massivos: aplicação as estrelas WCs e LBVs, Ph.D. Thesis

Machado, M. 1999, in Wolf-Rayet Phenomena in Massive Stars and Starburst Galaxies, Proc. IAU Symp. 193, ed. K. A. Van Der Hucht, G. Koenigsberger, \& P. R. J. Eenens (Dordrecht; Kluwer), 223
Merrill, P. W., \& Burwell, C. G. 1949, ApJ, 110, 387

Mihalas, D., \& Stone, M. E. 1968, ApJ, 151, 293

Schaller, G., Meynet, G., \& Maeder, A. 1992, A\&AS, 96, 269

Sheikina, A., Miroshnichenko, A., \& Corporon, P. 2000, The Be phenomenon in Early-Type Stars, IAU Colloquium 175, in press

Stee, Ph., Araújo, F. X., Vakili, F., et al. 1995, A\&A, 300, 219 Szeifert, Th., Humphreys, R. M., Davidson, K., et al. 1996, A\&A, 314, 131

Thé, P. S., de Winter, D., \& Pérez, M. R. 1994, A\&AS, 104, 315

Valenti, J. A., Johns-Krull, C. M., \& Linsky, J. L. 2000, ApJS, 129,399

Zickgraf, F.-J., Wolf, B., Stahl, O., Leithere, C., \& Appenzeller, I. $1986, \mathrm{~A} \& \mathrm{~A}, 163,119$ 